\title{
Expression of Cancer-Testis Antigens in Stem Cells: Is it a Potential Drawback or an Advantage in Cancer Immunotherapy
}

\author{
Soudeh Ghafouri-Fard
}

\begin{abstract}
Cancer-testis antigens (CTAs) are a group of tumor associated antigens with a restricted expression pattern in normal gametogenic tissues but expression in a broad range of malignancies. Their expression pattern has made them potential targets for immunotherapy. However, expression of some of these antigens has been demonstrated in normal stem cells as well as cancer stem cells (CSCs). As CSCs have been shown to be sources of metastasis and tumor recurrence, novel therapies are being focused on their eradication. On the other hand, CTA expression in normal stem cells raises the possibility that CTA based immunotherapies cause side effects in normal tissues.
\end{abstract}

Keywords: Cancer-testis antigen - cancer stem cell - immunotherapy

Asian Pac J Cancer Prev, 16 (7), 3079-3081

\section{Introduction}

Cancer-testis antigens (CTAs) are a group of tumor associated antigens whose expression has been demonstrated at both mRNA and protein levels in different cancer cell lines and tissues, along with normal testis, ovary and trophoblast (Ghafouri-Fard et al., 2010a; Ghafouri-Fard et al., 2010b; Dianatpour et al., 2012; Ghafouri-Fard and Modarressi, 2012; Ghafouri-Fard et al., 2012b). In addition, their expression has been demonstrated in various types of stem cells including mesenchymal stem cells (MSCs) of different adult and fetal tissues (Cronwright et al., 2005; Saldanha-Araujo et al., 2010), CD34+ hematopoietic stem cells (Steinbach et al., 2002) and embryonic stem cell lines (Lifantseva et al., 2011).

Although the function of all CTAs has not been defined yet, they are thought to contribute in early phases of embryonic development and in stem cell self-renewal. Heterogeneous expression of these antigens in tumor tissues along with other evidences have implied that expression of these antigens in tumor tissues is limited to cells that have stem cell properties (Ghafouri-Fard and Modarressi, 2009; Ghafouri-Fard et al., 2012a).

\section{Expression of CTAs in Cancer Stem Cells}

As suggested by CSC model of tumorigenesis cancers are mainly driven by a small population of stem cells which similar to normal tissue stem cells are capable of self-renewal (Tabarestani and Ghafouri-Fard, 2012). A recent study has demonstrated preferential expression of a significant number of cancer-testis (CT) genes in cancer stem cells (CSCs)/cancer-initiating cells (CICs), hence suggested a new sub-category of CT genes named cancer/ testis/stem genes (Yamada et al., 2013). Another study in glioma cell lines and tissues has shown common and high expression of CTA genes in cancer stem cells compared with differentiated cells. Furthermore, epigenetic studies of promoter regions of CTA genes in cancer stem cells have revealed hypomethylation and high histone acetylation compared with differentiated cells (Yawata et al., 2010).

\section{CTA Applications in Immunotherapy}

The existence of blood-testis barrier (also called Sertoli cell barrier) and the lack of HLA class I expression in the germ cells have made testis an immune-privileged site which has provided scientific basis for the recruitment of CTAs in immunotherapeutic approaches (GhafouriFard, 2012b). Currently, tens of clinical trials are being conducted in cancer patients using CTAs and some of them have shown promising results. Expression of CT genes in CSCs may provide special targets for treatment of cancer recurrences and metastasis (Ghafouri-Fard, 2012a; 
Soudeh Ghafouri-Fard

Ghafouri-Fard and Modarressi, 2012). CTA genes have been suggested as suitable targets for immunotherapy against cancer stem cells in some tumor types (Yawata et al., 2010). However, the potential off-target effects on MSCs should be considered in such therapies (SaldanhaAraujo et al., 2010)

\section{Biological Roles of MSCs}

MSCs are multipotent stromal cells with ability to differentiate into a range of cell types, such as osteoblasts, chondrocytes and adipocytes. Historically, they were first found in bone marrow, but their presence was later shown in almost all adult and fetal human tissues (Covas et al., 2008). The most relevant function of MSCs in immunotherapeutic approaches is their potent immune suppressive function. The immunomodulatory function of MSCs has been demonstrated to be performed via inhibition of many immunogenic processes such as $\mathrm{T}$ cell and NK cell proliferation, B cells and dendritic cells (DCs) maturation and cytotoxic $\mathrm{T}$ cells generation, along with induction of regulatory T cells (Tregs) (De Miguel et al., 2012). MSC-induced tolerance has been shown to be useful in allogeneic transplantations to decrease the incidence of graft-versus-host disease. In addition, MSCs are genetically engineered to express molecules acting against the autoimmune process (Jorgensen et al., 2003).

\section{Conclusion}

CTA based immunotherapeutic approaches has been considered to have the advantage of decreasing tumor burden with the minimum side effects in normal tissues which is provided by limited expression pattern of such antigens in normal tissues. Additional findings of their expression in CSCs have increased the hope that CTA based therapies can eradicate CSCs. However, recent findings have indicated that some of them are expressed in normal stem cells. NY-ESO-1 is among CTAs whose expression has been demonstrated in MSCs (Cronwright et al., 2005). This tumor associated antigen has been applied in several clinical trials with promising results in some of them. Despite its expression in normal MSCs, NY-ESO-1 based immunotherapies have been well tolerated which has been attributed to the difference between the pattern of NY-ESO-1 expression in normal and cancerous tissues (Hayes et al., 2014). As MSCs are considered to have immunomedulatory effects, the potential effect of CTAbased immunotherapies on these cells may be useful in mounting immune response. Considering the fact that tumor-driven immune evasion is mainly exerted via Tregs, this population of $\mathrm{T}$ cells represents ideal targets for testing new anticancer treatment modalities (Curiel, 2007). Future studies can evaluate the effect of MSC inhibition as well as Treg inhibition on the outcome of cancer immunotherapy. However, if immunotherapeutic approaches targeted against a certain CTA cause adverse off-target effects in tissues containing MSCs, other CTAs with no or lower expression in MSCs might be considered for substitute employment in clinical trials.

\section{References}

Covas DT, Panepucci RA, Fontes AM, et al (2008). Multipotent mesenchymal stromal cells obtained from diverse human tissues share functional properties and gene-expression profile with CD146+ perivascular cells and fibroblasts. Exp Hematol, 36, 642-54.

Cronwright G, Le Blanc K, Gotherstrom C, et al (2005). Cancer/ testis antigen expression in human mesenchymal stem cells: down-regulation of SSX impairs cell migration and matrix metalloproteinase 2 expression. Cancer Res, 65, 2207-15.

Curiel TJ (2007). Tregs and rethinking cancer immunotherapy. J Clin Invest, 117, 1167-74.

De Miguel MP, Fuentes-Julian S, Blazquez-Martinez A, et al (2012). Immunosuppressive properties of mesenchymal stem cells: advances and applications. Curr Mol Med, 12, 574-91.

Dianatpour M, Mehdipour P, Nayernia K, et al (2012). Expression of testis specific Genes TSGA10, TEX101 and ODF3 in breast cancer. Iran Red Crescent Med J, 14, 722-6.

Ghafouri-Fard S (2012a). Are cancer-testis antigens cancer stem cell markers. Single Cell Biol, 1, 104.

Ghafouri-Fard S (2012b). Immunotherapy in nonmelanoma skin cancer. Immunotherapy, 4, 499-510.

Ghafouri-Fard S, Abbasi A, Moslehi H, et al (2010). Elevated expression levels of testis-specific genes TEX101 and SPATA19 in basal cell carcinoma and their correlation with clinical and pathological features. Br J Dermatol, 162, 772-9.

Ghafouri-Fard S, Abdollahi DZ, Omrani M, et al (2012). shRNA mediated RHOXF1 silencing influences expression of BCL2 but not CASP8 in MCF-7 and MDA-MB-231 cell lines. Asian Pac J Cancer Prev, 13, 5865-9.

Ghafouri-Fard S, Modarressi MH (2009). Cancer-testis antigens: potential targets for cancer immunotherapy. Arch Iran Med, 12, 395-404.

Ghafouri-Fard S, Modarressi MH (2012). Expression of cancer-testis genes in brain tumors: implications for cancer immunotherapy. Immunotherapy, 4, 59-75.

Ghafouri-Fard S, Modarressi MH, Yazarloo F (2012b). Expression of testis-specific genes, TEX101 and ODF4, in chronic myeloid leukemia and evaluation of TEX101 immunogenicity. Ann Saudi Med, 32, 256-61.

Ghafouri-Fard S, Ousati Ashtiani Z, Sabah Golian B, et al (2010b). Expression of two testis-specific genes, SPATA19 and LEMD1, in prostate cancer. Arch Med Res, 41, 195-200.

Hayes SJ, Hng KN, Clark P, et al (2014). Immunohistochemical assessment of NY-ESO-1 expression in esophageal adenocarcinoma resection specimens. World J Gastroenterol, 20, 4011-6.

Jorgensen C, Djouad F, Apparailly F, et al (2003). Engineering mesenchymal stem cells for immunotherapy. Gene Ther, 10, 928-31.

Lifantseva N, Koltsova A, Krylova T, et al (2011). Expression patterns of cancer-testis antigens in human embryonic stem cells and their cell derivatives indicate lineage tracks. Stem cells international, 2011.

Saldanha-Araujo F, Haddad R, Zanette DL, et al (2010). Cancer/ Testis antigen expression on mesenchymal stem cells isolated from different tissues. Anticancer Res, 30, 5023-7.

Steinbach D, Hermann J, Viehmann S, et al (2002). Clinical implications of PRAME gene expression in childhood acute myeloid leukemia. Cancer Genetics Cytogenetics, 133, 118-23.

Tabarestani S, Ghafouri-Fard S (2012). Cancer stem cells and response to therapy. Asian Pac J Cancer Prev, 13, 5951-8.

Yamada R, Takahashi A, Torigoe T, et al (2013). Preferential expression of cancer/testis genes in cancer stem-like cells: proposal of a novel sub-category, cancer/testis/stem gene. 
Tissue Antigens, 81, 428-34.

Yawata T, Nakai E, Park KC, et al (2010). Enhanced expression of cancer testis antigen genes in glioma stem cells. Mol Carcinog, 49, 532-44 